\title{
Modern methods for investigating the stability of a pitching floating platform wind turbine
}

\author{
Matthew Lennie, David Marten, George Pechlivanoglou, Christian Navid Nayeri, and \\ Christian Oliver Paschereit \\ Chair of Fluid Dynamics, Hermann-Föttinger-Institut, Technische Universität Berlin, \\ Müller-Breslau-Str. 8, 10623 Berlin, Germany \\ Correspondence: Matthew Lennie (matthew.lennie@tu-berlin.de)
}

Received: 15 December 2016 - Discussion started: 21 December 2016

Revised: 18 September 2017 - Accepted: 1 November 2017 - Published: 22 December 2017

\begin{abstract}
The QBlade implementation of the lifting-line free vortex wake (LLFVW) method was tested in conditions analogous to floating platform motion. Comparisons against two independent test cases using a variety of simulation methods show good agreement in thrust forces, rotor power, blade forces and rotor plane induction. Along with the many verifications already undertaken in the literature, it seems that the code performs solidly even in these challenging cases. Further to this, the key steps are presented from a new formulation of the instantaneous aerodynamic thrust damping of a wind turbine rotor. A test case with harmonic platform motion and collective blade pitch is used to demonstrate how combining such tools can lead to a better understanding of aeroelastic stability. A second case demonstrates a non-harmonic blade pitch manoeuvre showing the versatility of the instantaneous damping method.
\end{abstract}

\section{Introduction}

The proliferation of large wind turbine rotors has been accompanied by the need for accurate and computationally inexpensive aeroelastic simulation tools. For aeroelastic simulations, the aerodynamics of the wind turbine are most typically calculated using methods based on blade element momentum (BEM). In the scenario of offshore wind, particularly when designing for floating platforms, the significant motion of the rotor leads to complicated aerodynamics. Sebastian and Lackner (2013) have made a convincing case that, even with secondary correction factors, floating platform wind turbine aerodynamics exceed the capabilities of BEM-based simulation methods. The main reason is that a floating platform wind turbine will dynamically pitch and yaw. As BEM does not explicitly solve the flow pattern of the wake, it is simply not possible to accurately represent such behaviour.

The lifting-line free vortex wake (LLFVW) method uses non-linear polar data ${ }^{1}$ to calculate the blade forces coupled

\footnotetext{
${ }^{1}$ Including viscous effects such as separation.
}

with a free vortex wake formulation and serves as a good method for simulating cases in which large rotor displacements and yaw misalignments occur (see Fig. 2). Recently, the implementation of an LLFVW code was completed and included in the QBlade wind turbine simulation code (Marten et al., 2015). Simultaneously to this study, the LLFVW solver was extended to include an unsteady aerodynamics model ${ }^{2}$ and coupled with the structural formulations of the FAST framework (Wendler et al., 2016; Saverin et al., 2016). In this paper, a comparison is made between the LLFVW code and existing literature comparisons in which higher-order aerodynamic simulation techniques were used, i.e. URANS CFD (Tran et al., 2014; Sebastian and Lackner, 2013). The comparisons and further test cases are made using the NREL $5 \mathrm{MW}$ reference turbine undergoing prescribed harmonic motion (see Fig. 1; Jonkman, 2013).

\footnotetext{
${ }^{2}$ LLFVW formulations inherently account for attached flow unsteadiness; the unsteady aerodynamic model mentioned here only includes terms for detached flow and leading edge vorticity. The details are given by Wendler et al. (2016).
} 
After the validation of the LLFVW code for simulations involving a moving rotor plane, the aerodynamic damping of the rotor is investigated. For this analysis, a new formulation is presented for the instantaneous aerodynamic damping of the fore-aft motion degree of freedom. The formulation is a modification of an existing formulation that was first presented by Bowles et al. (2014) and Corke and Thomas (2015) and later applied by Lennie et al. (2016) to an airfoil with microtabs. For the first time, this new formulation makes it possible to look at the aerodynamic damping throughout the pitch cycle of the wind turbine as opposed to the traditional approach in which only cycle-averaged values are inspected. Such a formulation is particularly useful for analysing aeroelastic instabilities for which limit cycle oscillations are present. Limit cycle oscillations will have cycleaveraged values that are neutral but could have occurrences of highly negative damping. Using this method on LLFVW data makes it possible to understand the aeroelastic thrust stability of the rotor without the heavy linearization of the aerodynamics applied in most stability analysis techniques. It is also a useful way of understanding the full effects of controller wind turbine interactions. An example will be presented showing the effect of collective blade pitch cycles during fore-aft motion of the rotor.

\section{Rotor motion}

For the scope of this paper, two varieties of prescribed motion are considered. The first variety, pitching, is the more realistic representation in which the rotor plane undergoes both pitch and linear translation (see Fig. 1). The second variety, fore-aft motion, assumes that the rotor plane pitching component is insignificant compared to the influence of the linear translation. Within the scope of this study investigating the total rotor thrust, the difference between the assumptions is assumed to be small. There may be applications for which this assumption is unsuitable. In the comparisons, the same magnitude and type of motion is used as in the literature so that no additional assumptions were introduced.

\section{Comparison cases}

The QBlade LLFVW implementation has been previously tested for a range of standard HAWT and VAWT cases as can be found in the existing literature (Marten et al., 2015; Saverin et al., 2016). When the wind turbine starts moving relative to the steady inflow, the wake will become distorted. In the case of a harmonic movement, the wake will display harmonic contractions and expansions (see Fig. 2) which induce velocity onto the rotor plane. The publications mentioned above focused on verifying the performance of the QBlade LLFVW under stationary conditions and cases with yaw. This means that the battery of verifications undertaken should be extended to include cases in which platform mo-

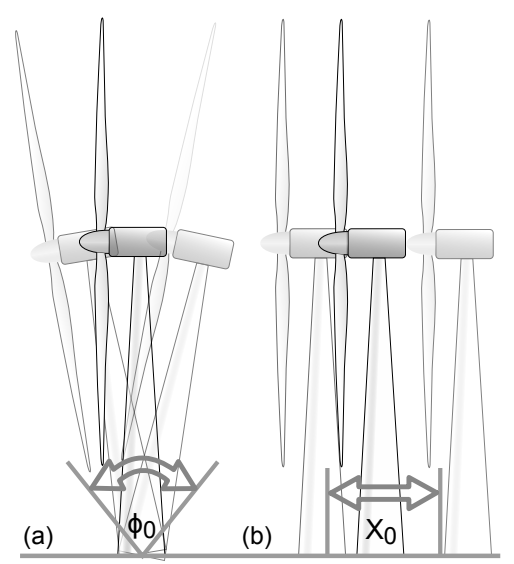

Figure 1. The two different assumed motions for the wind turbine: (a) wind turbine pitching and (b) wind turbine fore-aft motion.

tion is present, thus ensuring that LLFVW techniques are a suitable approach for floating platform wind turbine aerodynamics. A number of comparison papers have been sought from the literature that test a horizontal axis wind turbine rotor in prescribed floating platform motion. For the scope of this paper, only rigid body motion will be considered. The comparison will be undertaken by replicating the simulations from the literature which used higher-order methods.

Two different papers were used as a basis for comparison, both investigating the NREL 5MW reference turbine (Jonkman, 2013). Tran et al. (2014) compare a number of techniques with virtual blade motion using multiple reference frames (CFD-MRF) and real rigid body blade motion (CFD-RBM). The highest-order simulation is a 3-D unsteady Reynolds-averaged Navier-Stokes (URANS) CFD simulation with a $k-\omega$ shear-stress transport turbulence model. The blade rigid body motion was achieved using an overset grid, which is described at length including a discussion of the mesh convergence. The actual CFD simulations were conducted using the commercial code Fluent ${ }^{\mathrm{TM}}$ with Star$\mathrm{CCM}+{ }^{\mathrm{TM}}$ for meshing. It appears from the presented information that the simulations should be high quality and within the limitations of URANS.

Tran et al. (2014) also compared their results against lower-order simulations using the unsteady blade element momentum method. The Tran et al. (2014) implementation of the unsteady blade element moment (UBEM) method was taken from Hansen (2008) with corrections for tip losses, wake unsteadiness and unsteady aerodynamics. This particular implementation took the platform motion into account by changing the relative inflow velocity. Further comparisons were made using modified versions of FAST (Jonkman, 2015) from the National Renewable Energy Laboratory; one comparison used a momentum balance for the wake solution (FAST-BEM) and the other used generalized dynamic wake (FAST-GDW). In both cases, the structural modes were 


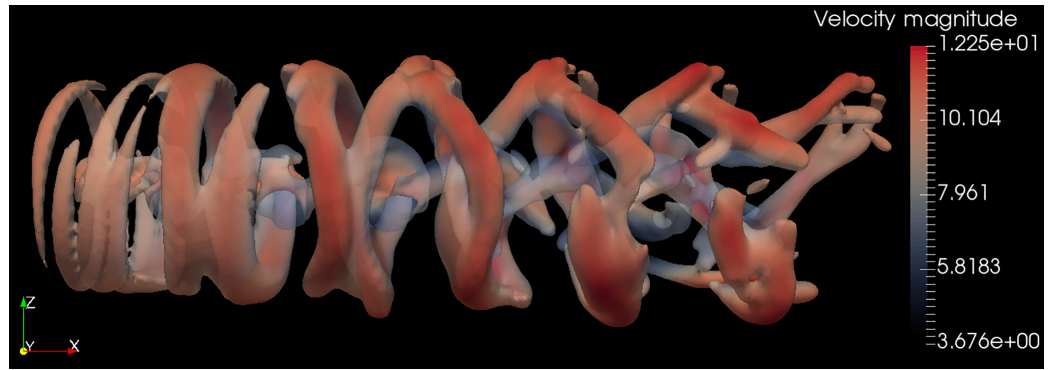

Figure 2. Snapshot of LLFVW simulation during pitching platform motion; vorticity isosurface of the wake coloured with velocity magnitude.

locked and no controller was used; for more complete details, see the original paper from Tran et al. (2014).

Tran et al. (2014) simulate the pitching of the wind turbine as shown in Fig. 1 (see the left-hand side). Two cases were simulated with platform pitching amplitudes of 1 and $4^{\circ}$ and a constant harmonic pitching frequency of $0.1 \mathrm{~Hz}$. The calculations were performed at a steady inflow speed of $11 \mathrm{~m} \mathrm{~s}^{-1}$ with a constant rotational speed of $12 \mathrm{rpm}$ and a constant blade pitch angle of $0^{\circ}$. From this paper it was possible to compare thrust, power and integrated blade forces.

The QBlade simulations were run with the same conditions as described above. The unsteady aerodynamic model from Wendler was enabled without vortex lift as the wind turbine is operating at near rated speed without yaw (Wendler et al., 2016). The standard NREL 5MW model (Jonkman, 2013) was set up according to the definition ${ }^{3}$.

The simulation settings can be found in Table 1. The FVLLW requires a number of input parameters which control the behaviour of the wake. The QBlade implementation of FVLLW lumps together wake elements in two stages in order to simulate a full-length wake without prohibitive computational costs. The wake age, full wake and fine wake parameters determine the positions at which the two stages of wake thinning occur and at which the wake is finally truncated. The parameters are described in terms of rotor revolutions to remove the dependency of the parameters on the tip speed ratio. The wake thin factor describes the extent to which the wake is thin. The initial vortex core radius is an important parameter for the de-singularization of the Biot-Savart equation. The turbulent vortex viscosity introduces diffusion to the wake. These parameters should be noted in attempts to reproduce the simulations performed in this paper. The full explanation of each of these parameters can be found in the paper from Marten et al. (2015).

The LLFVW (Fig. 2) shows moderately good agreement for all cases, which can be seen in Figs. 3, 4 and 5. It is interesting to note that steady and even unsteady BEM simulations, when compared to CFD or LLFVW results, underpredict the magnitude of the load cycle in most cases. The

\footnotetext{
${ }^{3}$ The standard 5MW project file is available for download with the standard QBlade package.
}

Table 1. QBlade FVLLW wake simulation settings.

\begin{tabular}{lr}
\hline Wake age in revolutions & 8 \\
Full wake in revolutions & 0.5 \\
Fine wake in revolutions & 4 \\
Wake thin factor & 2 \\
Initial vortex core radius & 0.20 \\
Turbulent vortex viscosity & 40 \\
Time step & $0.1 \mathrm{~s}$ \\
\hline
\end{tabular}

three classes of simulations compared all face limitations. The LLFVW and BEM cases both rely on 2-D polar data which are sensitive to measurement or simulation settings like wind tunnel turbulence or turbulence model. Lennie et al. (2015) and Eisele et al. (2013) have shown that the quality of the 2-D polar data is critical for power and load prediction. For the BEM-based methods, it is expected that the empirical corrections will struggle to represent the complicated fore-aft motion. The CFD solutions are based on unsteady Reynolds-averaged Navier-Stokes (URANS) equations. After conducting thorough verification and validations of multiple URANS solvers with multiple turbulence closure models, Rumsey showed that URANS-based models have a very limited ability to model cases with separation (Stangfeld et al., 2015; Rumsey, 2016). Furthermore, the tendency of URANS codes to smear vorticity will cause errors in the wake induction for cases in which the blades are modelled. With each of the simulation methods facing some sort of limitation, it is difficult to choose one method as the baseline or "most" accurate. Nonetheless, integrated blade forces agree well for the CFD from Tran et al. (2014) and the LLFVW (see Fig. 5). Discrepancies in thrust and power can be seen in Figs. 3 and 4 , but they are of a reasonable magnitude.

In the publication chosen for a second comparison, Vaal et al. (2014) use a moving actuator disc CFD hybrid method, which allows for a good comparison of the unsteady wake induction between CFD and the LLFVW. The moving actuator disc model essentially places a moveable actuator disc into a CFD simulation (implemented in Fluent ${ }^{\mathrm{TM}}$ ). In practice this means that the actuator disc acts as a volume force onto 

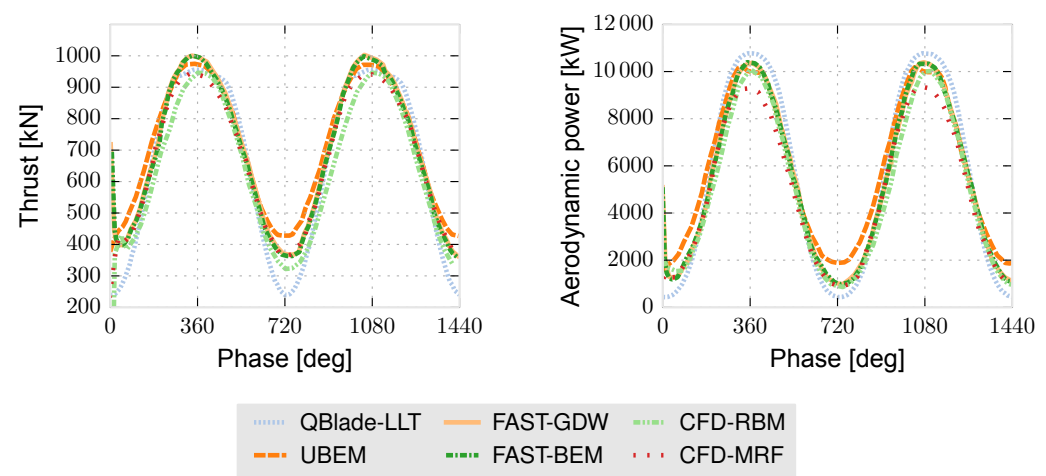

Figure 3. Thrust and power over phase angle for pitching platform motion ( $4^{\circ}$ pitch amplitude). Comparison case: Tran et al. (2014).
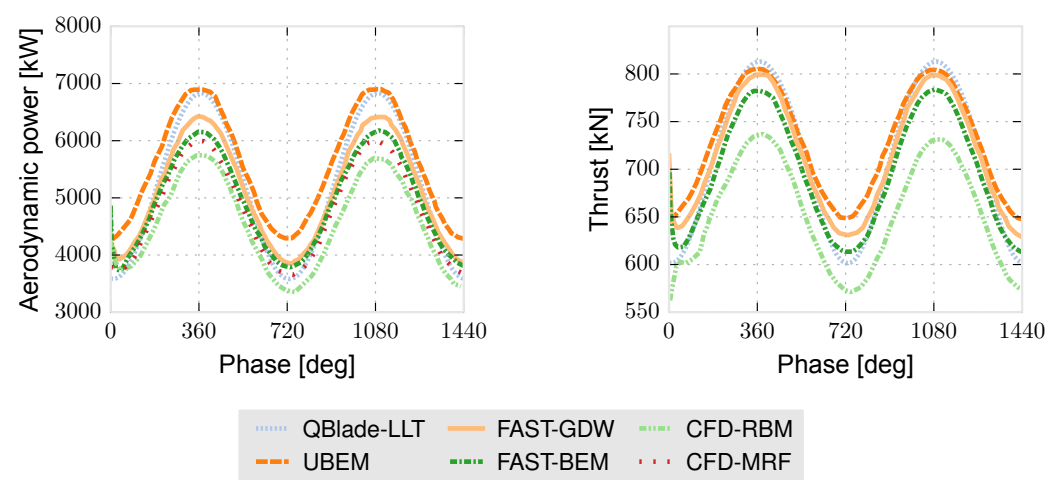

Figure 4. Thrust and power over phase angle for pitching platform motion ( $1^{\circ}$ pitch amplitude). Comparison case: Tran et al. (2014).

the surrounding cells. It is argued by Vaal et al. (2014) that, because this method explicitly solves the wake rather than relying on simplified relations, the method should be more robust than commonly used methods such as the Pitt-Peters model (Pitt and Peters, 1983) or the Stig Øye model (Hansen, 2008). Vaal et al. (2014) present a number of investigations into the relative performance of the models; for this paper, the rotor plane induction is the most interesting to compare.

Vaal et al. (2014) undertook a sensitivity study showing the wake velocity before and after the rotor at different phases for different operating conditions. From this study, the authors choose the largest amplitude $(16 \mathrm{~m})$ of fore-aft movement (right-hand side of Fig. 1). The fore-aft motion was harmonic with a frequency of $0.08 \mathrm{~Hz}$; the inflow speed was $11.2 \mathrm{~m} \mathrm{~s}^{-1}$, the blade pitch was $0^{\circ}$ and the rotor speed was a constant $0.2 \mathrm{~Hz}$. Vaal et al. (2014) allowed several oscillations to pass in order to let the wake effects develop. The grid extended 10 rotor diameters upstream and downstream. It appears that the approach and settings used by Vaal et al. (2014) provide good-quality results for comparison.

The QBlade simulations were conducted again using the settings stated above, with prescribed linear rotor plane movement. Like Vaal et al. (2014), a number of oscillations were simulated before finally extracting the data. The comparison was made at the exact rotor plane for which the axial velocity could be sampled over an area determined by the rotor radius. There was not enough information provided by Vaal et al. (2014) to ensure the consistency of the sampling area for the induced velocity. In the context of a wake with expansion and contraction occurring, the assumptions have a distinct effect on the induction results. Therefore, no upstream or downstream comparisons were attempted. A rotor plane axial velocity field snapshot was taken at equally spaced points within the cycle. The results in Fig. 6 show that the rotor plane induction for the two methods matches well over the four snapshots. It therefore seems that there is a good agreement between the two methods for the most challenging test case presented by Vaal et al. (2014).

From the two verifications performed here it seems that the QBlade LLFVW simulation model produces results that are comparable to other higher-order or hybrid methods. These results and the results already published give a high degree of confidence in the simulation tool's ability to model wind turbines undergoing platform motion. 

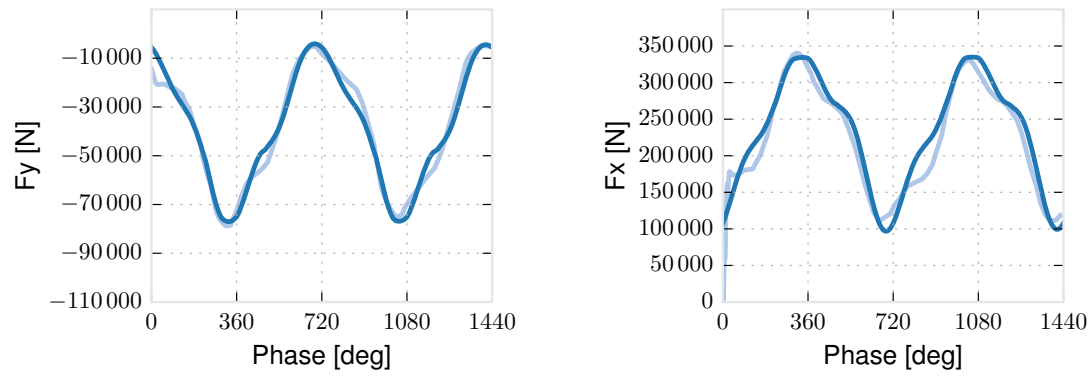

Blade 1 - QBlade LLT Blade 1

Figure 5. Integrated blade forces at blade root for pitching platform motion (4 pitch amplitude). Comparison case: Tran et al. (2014).

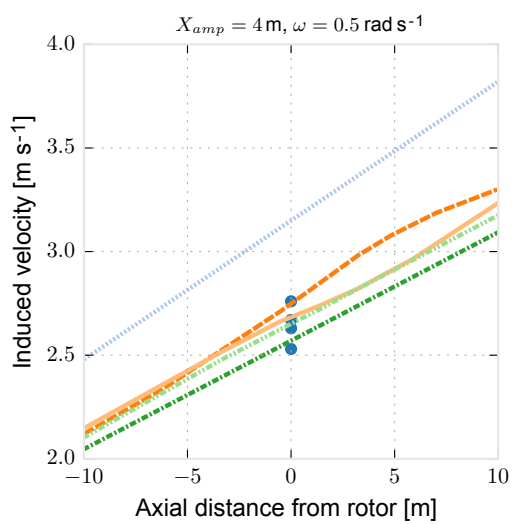

Steady $=0.00 \mathrm{~T}-0.25 \mathrm{~T}=0.50 \mathrm{~T}$

0.75T $\bullet \bullet$ QBlade LLT

Figure 6. Induction plot compared to moving actuator disc CFD hybrid from Vaal et al. (2014).

\section{Using the Hilbert transform method to obtain instantaneous aerodynamic damping of a translating rotor}

The following section briefly outlines a reformulation of the instantaneous damping calculation method outlined by Bowles et al. (2014) and Corke and Thomas (2015). The original reference by Bowles et al. (2014) describes the original method in complete detail, and Lennie et al. (2016) provide an application of the method to an airfoil with microtabs and gurney flaps. In the reformulation described here, the instantaneous damping calculation is applied to the fore-aft motion of the whole rotor, a situation particularly interesting for floating platform wind turbines. It is assumed for this paper that a small pitch angle means that the linear motion will have a greater effect on the wake than the pitching of the rotor plane.

\subsection{Cycle harmonic damping}

Before setting out the derivation of the instantaneous damping coefficient it is first essential to set out the cycle total aerodynamic damping. The approach taken follows the derivation provided by Carta and Niebanck (1969) but for a rotor undergoing fore-aft motion rather than a pitching airfoil section. The authors would like to clearly acknowledge that the following derivation is a modification of existing concepts rather than a completely new derivation.

\subsubsection{Linear harmonic system in a vacuum}

To begin the derivation, let us start with the homogeneous equation of the wind turbine oscillating in linear fore-aft motion (denoted as $x$ ):

$m \ddot{x}^{*}+c \dot{x}^{*}(t)+k x^{*}(t)=0$,

where we make an assumption of harmonic motion thus taking

$x^{*}(t)=x_{0} e^{i \omega t}$,

which then gives

$\dot{x}^{*}(t)=i \omega x_{0} e^{i \omega t}$,
$\ddot{x}^{*}(t)=-\omega^{2} x_{0} e^{i \omega t}$. 
Substituting these results into the equation of motion then gives

$(-\omega^{2} m-\overbrace{i \omega c}^{\mathbb{C}}+k) x^{*}(t)=0$.

As highlighted by Carta and Niebanck (1969), the damping terms are contained within the imaginary term of the equation of motion. For similar linear systems, the damping should be contained within the imaginary terms of the differential equation.

\subsubsection{Linear harmonic system in air}

Now by introducing the aerodynamic terms into the equation we arrive at the particular equation of

$(-\omega^{2} m-\overbrace{i \omega c}^{\mathbb{C}}+k) x^{*}(t)=T_{\mathrm{U}}^{*}$.

Still following the logical steps set down by Carta and Niebanck (1969), the unsteady thrust force can be written as

$T_{\mathrm{U}}^{*}=T_{1} \ddot{x}^{*}+T_{2}^{*} \dot{x}^{*}+T_{3}^{*} x^{*}$,

where

$T_{2}, T_{3} \in \mathbb{C}$,

but

$T_{1} \in \mathbb{R}$

due to the rationale that $T_{1}$ represents the apparent mass terms of the system which are identified in terms of the instantaneous reaction forces of an impermeable disc in still air. Instantaneous reaction forces are in phase with the acceleration and therefore real. A similar rationale was used in the SDOF torsional airfoil oscillator formulation by Carta and Niebanck (1969), Bisplinghoff et al. (2013) and Scanlan and Rosenbaum (1951), this time by directly comparing to the Theodorsen theory (Theodorsen, 1935) in which a wellknown distinction is made between the real and imaginary parts of the Bessel function. By substituting in the Cartesian forms of the thrust force

$T_{2}=T_{2 \mathrm{R}}+i T_{2 \mathrm{I}}$,

$T_{3}=T_{3 \mathrm{R}}+i T_{3 \mathrm{I}}$,

we arrive at the following equation:

$$
\begin{gathered}
\left(m-T_{1}\right) \ddot{x}^{*}+\left(c-T_{2 \mathrm{R}}-i T_{2 \mathrm{I}}\right) \dot{x}^{*} \\
\quad+\left(k-T_{3 \mathrm{R}}-i T_{3 \mathrm{I}}\right) x^{*}=0 .
\end{gathered}
$$

By assuming harmonic motion and collecting real and imaginary terms, the equation is reduced to

$$
\begin{gathered}
{\left[\left(-\omega^{2}\left(m-T_{1}\right)+\omega T_{2 \mathrm{I}}+k-T_{3 \mathrm{R}}\right)\right.} \\
\left.\quad+i\left(\omega\left(c-T_{2 \mathrm{R}}\right)-T_{3 \mathrm{I}}\right)\right] x^{*}=0 .
\end{gathered}
$$

By eliminating the mechanical damping terms, which are the terms present in a vacuum, the aerodynamic damping can be shown as

$\xi=-\omega T_{2 \mathrm{R}}-T_{3 \mathrm{I}}$.

This result will form a key step in the next decomposition.

\subsubsection{Work done by a rotor in fore-aft motion}

Let us now take a different decomposition of the thrust force into its constituent steady and unsteady parts ${ }^{4}$.

$T_{\mathrm{TOTAL}}=T_{\mathrm{MEAN}}+T_{\mathrm{UR}} \cos \omega t+T_{\mathrm{UI}} \sin \omega t$

Two options exist for the normalization of the thrust force: the freestream velocity or with the inflow velocity thus accounting for the rotor movement. The former assumption simply implies that the unsteady coefficient will contain the freestream effects for the expected velocity ratios expected for wind turbine pitching movement and freestream velocities. This may cause some peculiarities in the appearance of the data similar to the lift coefficient overshoots seen by Müller-Vahl (2015) and Strangfeld (2015) in unsteady airfoil wind tunnel measurements. Nonetheless, the freestream velocity is taken as convention, meaning that the unsteady features will be wrapped up into the unsteady thrust coefficient.

This gives the coefficient form

$C_{\mathrm{TTOTAL}}=C_{\mathrm{TMEAN}}+C_{\mathrm{TUR}} \cos \omega t+C_{\mathrm{TUI}} \sin \omega t$.

The work performed over one cycle of rotor fore and aft motion can be given as

$W_{\mathrm{T}}=\oint T_{\mathrm{TOTAL}} \mathrm{d} x$

or in coefficient form

$C_{\mathrm{WT}}=\oint C_{\mathrm{TTOTAL}} \mathrm{d} x$.

Here the differential operator can be switched

$\mathrm{d} x=x_{0} \sin \omega t \mathrm{~d} \omega t$,

and the integral range can be set from $0<\omega t<-2 \pi$ to capture a single cycle, finally giving

$$
\begin{gathered}
C_{\mathrm{WT}}=-\int_{0}^{2 \pi}\left[C_{\mathrm{MEAN}}+C_{\mathrm{TUR}} \cos \omega t\right. \\
\left.+C_{\mathrm{TUI}} \sin \omega t\right] x_{0} \sin \omega t \mathrm{~d} \omega t .
\end{gathered}
$$

By assuming that the thrust force will be simple harmonic (or deviate minimally), evaluating the integral shows that the

\footnotetext{
${ }^{4} T_{\text {Unsteady }}$ is hereafter abbreviated $T_{\mathrm{U}}$.
} 
real unsteady term and the mean terms are both eliminated during the integration, leaving

$C_{\mathrm{WT}}=\pi x_{0} C_{\mathrm{TUI}}$.

This result can also be represented in the form

$C_{\mathrm{WT}}=\pi x_{0} C_{\mathrm{TUI}}=x_{0} C_{T_{0}} \sin \phi$.

This result is the second of the building blocks required to extract the aerodynamic damping from the measurement or simulation data.

\subsubsection{The third decomposition of the thrust force}

Let us now inspect the unsteady thrust force terms further. If we assume the thrust to be a sinusoidal time-dependent function, the unsteady thrust force can be given in the Cartesian form

$T_{\mathrm{U}}=T_{\mathrm{UR}}+i T_{\mathrm{UI}}$.

For a prescribed motion system, the earlier homogeneous equation (Eq. 13) can be written as a particular equation, thus giving

$$
\begin{aligned}
T_{\mathrm{U}} & =T_{\mathrm{UR}}+i T_{\mathrm{UI}}=\left[\left(-\omega^{2}\left(m-T_{1}\right)+\omega T_{2 \mathrm{I}}+K-T_{3 \mathrm{R}}\right)\right. \\
& \left.+i\left(\omega\left(C-T_{2 \mathrm{R}}\right)-T_{3 \mathrm{I}}\right)\right] x .
\end{aligned}
$$

By equating real and complex terms we get

$T_{\mathrm{UI}}=\left[\left(\omega\left(c-T_{2 \mathrm{R}}\right)-T_{3 \mathrm{I}}\right)\right] x$.

This result provides the key to extracting the aerodynamic damping coefficient from the thrust data. From earlier we know that

$\xi=\left(-\omega T_{2 \mathrm{R}}-T_{3 \mathrm{I}}\right)$.

These two equations can be related through

$\xi=-\frac{\mathrm{d} T_{\mathrm{UI}}}{\mathrm{d} x}$

or written in the coefficient form

$\Xi=-\frac{\mathrm{d} C_{\mathrm{TUI}}}{\mathrm{d} x}$.

Using the earlier result of

$C_{\mathrm{WT}}=\pi x_{0} C_{\mathrm{TUI}}=x_{0} C_{\mathrm{T} 0} \sin \phi$,

we can finally arrive at the conclusion that

$\Xi_{\text {CYCLE }}=\frac{\mathrm{d} \frac{C_{\mathrm{WT}}}{\pi x_{0}}}{\mathrm{~d} x}=-\frac{1}{\pi x_{0}^{2}} \oint C_{\mathrm{TTOTAL}} \mathrm{d} x$.

This equation will form the basis of checking whether the instantaneous equation formulation is correct. In practice it also provides a useful debugging tool for the code implementation.

\subsection{Instantaneous damping derivation}

In most formulations, certainly as shown above, only a cycleaveraged value of aerodynamic damping is found. Bowles et al. (2014) and Corke and Thomas (2015) provided a breakthrough on this front by using the Hilbert transform to get an estimate of the magnitude and phase of a signal. If we inspect the following equation from the earlier whole-cycle derivation, we may already see the general direction that such a method would follow.

$C_{\mathrm{WT}}=x_{0} T_{0} \sin \phi$

The instantaneous damping derivation begins with yet another form of the basic equation of motion for the prescribed fore-aft motion of a wind turbine rotor. The prescribed motion leaves only the aerodynamic forces,

$m_{t} \ddot{x}+h^{*}(t) \dot{x}(t)+\kappa^{*} x(t)=T(t)$,

into which we can insert the apparent mass or inertia of air (Pitt and Peters, 1983), ${ }^{5}$

$m_{\text {tair }}=\frac{8}{3} \rho R^{3}$,

and then the complex damping and stiffness terms can be described in polar form as

$h^{*}=h_{r}+i h_{\mathrm{I}}=\bar{h} e^{i \gamma_{1}}$,

$\kappa^{*}=\kappa_{r}+i \kappa_{\mathrm{I}}=\bar{\kappa} e^{i \gamma_{2}}$.

Thus we arrive at

$\frac{8}{3} \rho R^{3} \ddot{x}+\bar{h} e^{i \gamma_{1}} \dot{x}+\bar{\kappa} e^{i \gamma_{2}} x=T$.

By introducing the natural frequency parameter $\omega_{0}$ as

$\omega_{0}=\sqrt{\frac{3 \bar{\kappa}}{8 \rho R^{3}}}$,

the equation reduces to

$\ddot{x}+2 h_{0} \omega_{0} e^{i \gamma_{1}} \dot{x}+\omega_{0}^{2} e^{i \gamma_{2}} x=\frac{3}{8 \rho R^{3}} T$.

Now taking the Hilbert transform of both sides of the equation results in

$\ddot{\mathcal{X}}+2 h_{0} \omega_{0} e^{i \gamma_{1}} \dot{\mathcal{X}}+\omega_{0}^{2} e^{i \gamma_{2}} \mathcal{X}=\frac{3 \mathcal{T}}{8 \rho R^{3}}$,

\footnotetext{
${ }^{5}$ The inertia is not used in this derivation and the apparent mass terms are actually cancelled out later. However, it is important to note that the apparent mass analogy can be made for a rotor. If that were not true, then the first term would also be complex and this derivation would be invalidated.
} 
where we replace the thrust and movement with their analytical signal counterparts

$\mathcal{X}=X+i \widetilde{X}=x_{\mathrm{amp}} e^{i \omega t}$,

$\mathcal{T}=T_{u}+i \widetilde{T}_{u}=A_{t}(t) e^{\phi(t)}$,

and equate the imaginary and real components

$$
\begin{aligned}
& -\omega^{2}-2 h_{0} \omega \sin \gamma_{1}+\omega_{0}^{2} \cos \gamma_{2}+i\left(2 h_{0} \omega \cos \gamma_{1}+\omega_{0}^{2} \sin \gamma_{2}\right) \\
& =\frac{3}{8 \rho R^{3} x_{\mathrm{Amp}}}\left(T_{u}+i \widetilde{T}_{u}\right) e^{-i \omega t} .
\end{aligned}
$$

Again, the imaginary components correspond to the damping of the system.

$$
\begin{aligned}
& 2 h_{0} \omega \cos \gamma_{1}+\omega_{0}^{2} \sin \gamma_{2} \\
& \quad=\frac{3}{8 \rho R^{3} x_{\mathrm{Amp}}}\left(\widetilde{T}_{u} \cos \omega t-T_{u} \sin \omega t\right) \\
& \quad=\frac{3 A_{T}(t)}{8 \rho R^{3} x_{\mathrm{Amp}}} \sin \overbrace{(\phi(t)-\omega t)}^{\psi}
\end{aligned}
$$

The left-hand term of this equation correlates with the damping of the system normalized by the apparent mass of the air using a combination of the equations set down by Carta and Niebanck (1969) and the normalization highlighted by Bowles et al. (2014) and Corke and Thomas (2015).

$\xi=\frac{A_{T}(t)}{x_{\mathrm{Amp}}} \sin \overbrace{(\phi(t)-\omega t)}^{\psi}$

Finally, we can normalize

$\Xi(t)=\frac{\xi}{P_{\mathrm{dyn}} A}=-\frac{A_{C_{t}}(t)}{x_{\mathrm{amp}}} \sin \psi(t)$,

where $\widetilde{C}_{t}(t)$ is given by the Hilbert-transformed thrust coefficient time series $C_{t}(t)$

$\widetilde{C}_{t}(t)=\mathcal{H}\left[C_{t}\right]=-\frac{1}{\pi} \mathcal{P} \int_{-\inf }^{\inf } \frac{C_{t}(\tau)}{\tau-t} \mathrm{~d} t$,

thus giving the analytical signal magnitude

$A_{C_{t}}=\sqrt{C_{t}^{2}+\widetilde{C}_{t}^{2}}$

and phase

$\phi(t)=\arg (Y(t))=\arg \left(C_{t}+\widetilde{C}_{t}\right)$,

which gives us the phase difference between the lift and the fore-aft motion,

$\psi(t)=\phi(t)-\omega t$, from the assumed motion

$$
X(t)=X_{0} e^{i \omega t} .
$$

The time-averaged damping then gives us the cycle damping:

$\Xi_{\mathrm{avg}}=-\frac{1}{T} \int_{0}^{T} \Xi(t) \mathrm{d} t$.

As previously undertaken by Bowles et al. (2014), Corke and Thomas (2015) and Lennie et al. (2016), the cycle-averaged damping formulation provided by Carta and Niebanck (1969) can be used as a comparison. For this derivation, the comparison will be against the formula derived earlier for the fore-aft motion of a rotor.

$\Xi=\frac{\mathrm{d} \frac{C_{\mathrm{WT}}}{\pi X_{0}}}{\mathrm{~d} x}=-\frac{1}{\pi X_{0}^{2}} \oint C_{\text {TTOTAL }} \mathrm{d} x$

Agreement between the two calculations provides a useful (although not completely "leak-proof") verification that the analytical signal is well conditioned and that no implementation errors are present. Verifications undertaken in Lennie et al. (2016) for the original formulation showed less than $<1 \%$ variation between the methods; Bowles et al. (2014) also remarked on the good agreement.

On the practical side, Hilbert transforms are intended to analyse narrowband signals. It was previously established in Lennie et al. (2016) that numerical or experimental noise does not cause problems for this formulation; therefore no signal filtering will be applied. Both original and phaseaveraged data were analysed, although only graphs of the phase-averaged data are presented in the paper. In the original time series, stochastic variations due to turbulent inflow are present in cases with turbulence. Otherwise after a few cycles the results converge to the phase mean.

\section{Demonstration case 1: collective blade pitch}

Having presented the analysis methods, it is possible to use these methods to investigate an example case of floating platform wind turbine aeroelasticity. A case was selected that should demonstrate more complicated thrust damping behaviour. The case chosen is harmonic collective pitch in the presence of platform translation. Further potential test cases for future work would include harmonic platform movement in combination with the following:

- yawed inflow;

- inflow turbulence;

- gusts or sudden changes in direction; 


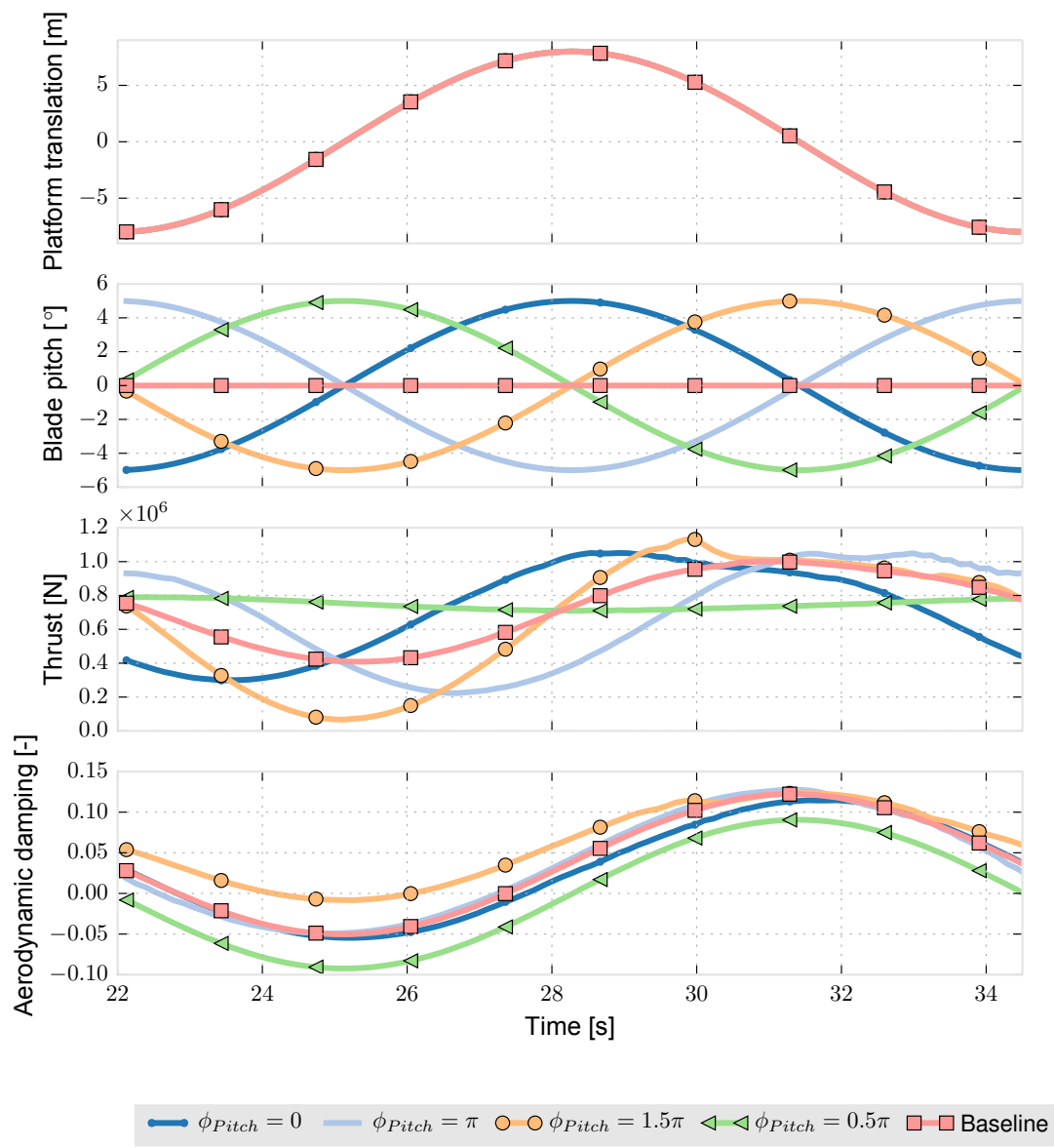

Figure 7. Collective pitch damping cycles.

- changes in airfoil performance through simulated active flow control;

- and/or non-synchronous pitch and platform movements.

Harmonic collective pitch in conjunction with platform movement is a complicated test case, but it is still simple enough to give a good demonstration of this particular tool chain. The collective pitch motion was prescribed using the formula

$\alpha=\alpha_{0} \sin \left(\omega t+\phi_{\text {pitch }}\right)$.

The test case settings are given in Table 2 .

The collective pitch cycle chosen is not a realistic control regime, but it was chosen to give a clear demonstration of the method. The LLFVW simulation was run for $60 \mathrm{~s}$ with a single cycle chosen for analysis after the initial wake effects had died out. The instantaneous damping was calculated from the thrust data using the method already discussed. As a verification the two cycle-averaged values were compared and had good agreement; the values are presented in Table 1.

The cycle-averaged aerodynamic damping values do in fact show that collective pitch has an effect. While thrust
Table 2. Demonstration case 1 simulation settings.

\begin{tabular}{lr}
\hline Rotor speed $(\mathrm{rpm})$ & 12.1 \\
$\alpha_{0}\left(^{\circ}\right)$ & 0.5 \\
$\phi_{\text {pitch }}(-)$ & $0,0.5 \pi, \pi, 1.5 \pi$ \\
Inflow velocity $\left(\mathrm{m} \mathrm{s}^{-1}\right)$ & 11.4 \\
$\omega_{\text {pitch }}, \omega_{\text {platform }}\left(\mathrm{rad} \mathrm{s}^{-1}\right)$ & 0.5 \\
\hline
\end{tabular}

forces tend to be positively damped (with this sign convention, that means good damping), we can see that the magnitude of the damping is altered. In Fig. 7, it is possible to follow the chain of logic that leads to these changes. In the thrust force sequences, it is possible to see that while there are some magnitude shifts, the more important feature is that the phase of the thrust force is shifted. This then manifests as changes to the aerodynamic damping.

A closer inspection reveals an interesting feature: a $0.5 \pi$ (green) phase shift of the pitching sequence leads to an almost constant thrust force. This may appear to be favourable to reduce the fatigue loads of the wind turbine. However, what has effectively happened is that there is no force in phase with the velocity of the movement and therefore no 


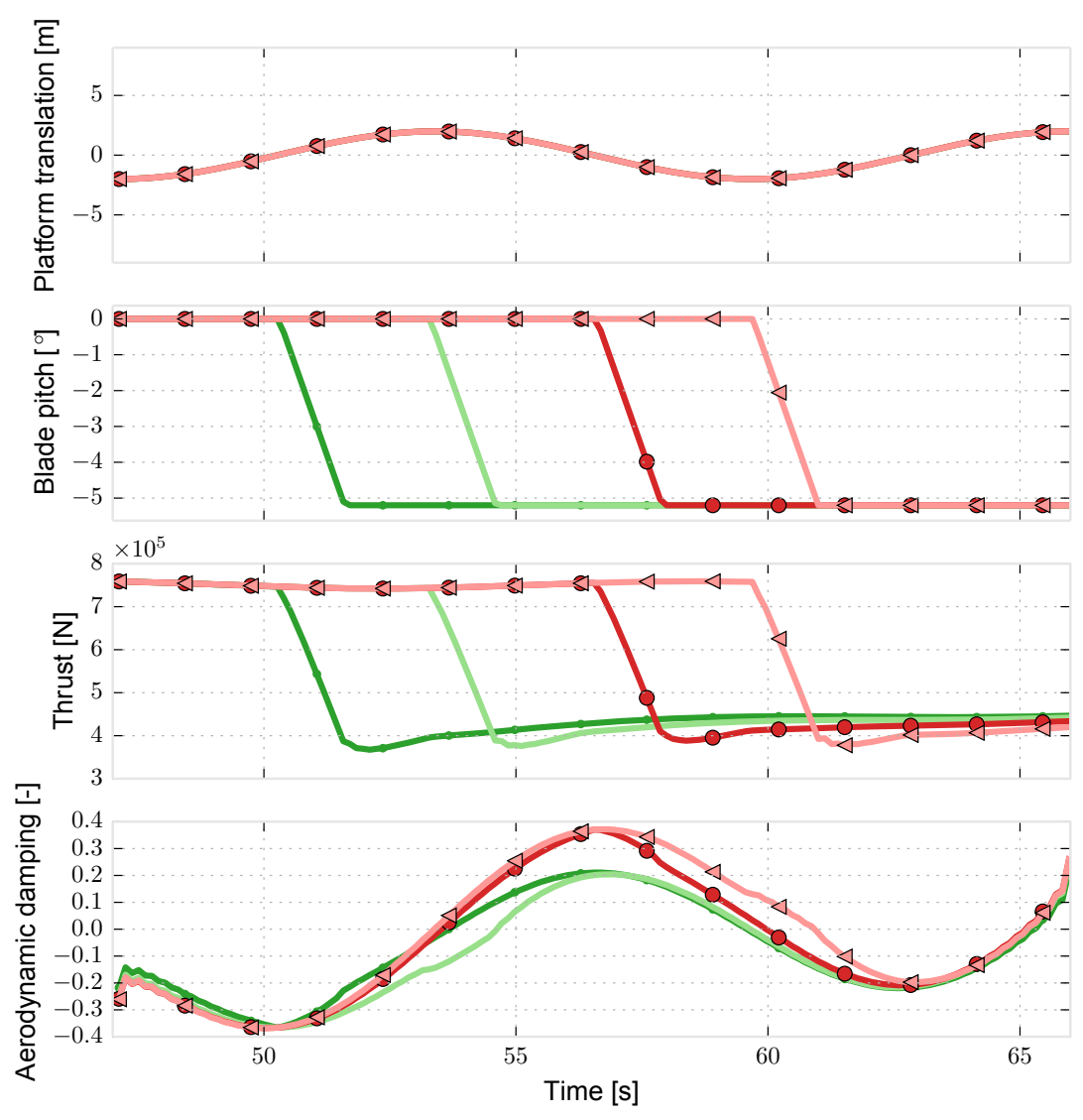

$$
\phi_{\text {Pitch }}=0.5 \pi-\phi_{\text {Pitch }}=\pi \stackrel{0}{\circ} \phi_{\text {Pitch }}=-0.5 \pi \triangleleft \triangleleft \phi_{\text {Pitch }}=0
$$

Figure 8. Negative blade pitch manoeuvre.

Table 3. Comparison of cycle damping values.

\begin{tabular}{lrrr}
\hline & $\begin{array}{r}\text { Averaged } \\
\text { instantaneous } \\
\text { damping }\end{array}$ & $\begin{array}{r}\text { Cycle- } \\
\text { averaged } \\
\text { damping }\end{array}$ & \\
\hline$\phi_{\text {Pitch }}=0$ & 0.030 & 0.031 & $3.5 \%$ \\
$\phi_{\text {Pitch }}=\pi$ & 0.036 & 0.038 & $3.5 \%$ \\
$\phi_{\text {Pitch }}=1.5 \pi$ & 0.060 & 0.062 & $3.5 \%$ \\
$\phi_{\text {Pitch }}=0.5 \pi$ & -0.001 & -0.001 & $3.5 \%$ \\
Baseline & 0.036 & 0.037 & $3.5 \%$ \\
\hline
\end{tabular}

complex term, thus resulting in slightly negative aerodynamic damping. In this case, the system would rely on the other sources of damping ${ }^{6}$ to reduce the amplitude of oscillation.

In the opposite case with a pitch phase shift of $1.5 \pi$ (yellow), the thrust force is more in phase with the velocity, and thus the opposing movement of the rotor is enhanced. The cycle-averaged damping reflects this with a stronger damping

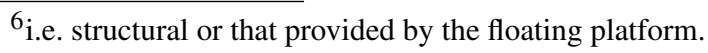

value. The instantaneous damping value starts to show some departure from a pure harmonic signal. This can be traced to the matching non-linearity in the thrust force, which could arise from rotor wake interactions; it is these effects that are difficult to account for in a cycle-averaged value. In the literature examples in which a pitching airfoil was examined (Bowles et al., 2014; Corke and Thomas, 2015; Lennie et al., 2016), the non-linearities were very strong due to dynamic stall and caused strong spikes in aerodynamic damping. In simulations in which sudden changes of operating conditions are present, the instantaneous damping method will highlight sudden drops in aerodynamic damping when they occur, even if they do not show up in the cycle-averaged values.

\section{Demonstration case 2: collective blade pitch manoeuvre}

The second case attempts to demonstrate one of the most useful aspects of the instantaneous damping approach. Again in this case, we will look at a collective blade pitch manoeuvre, but this time the pitching will not be periodic. This highlights 

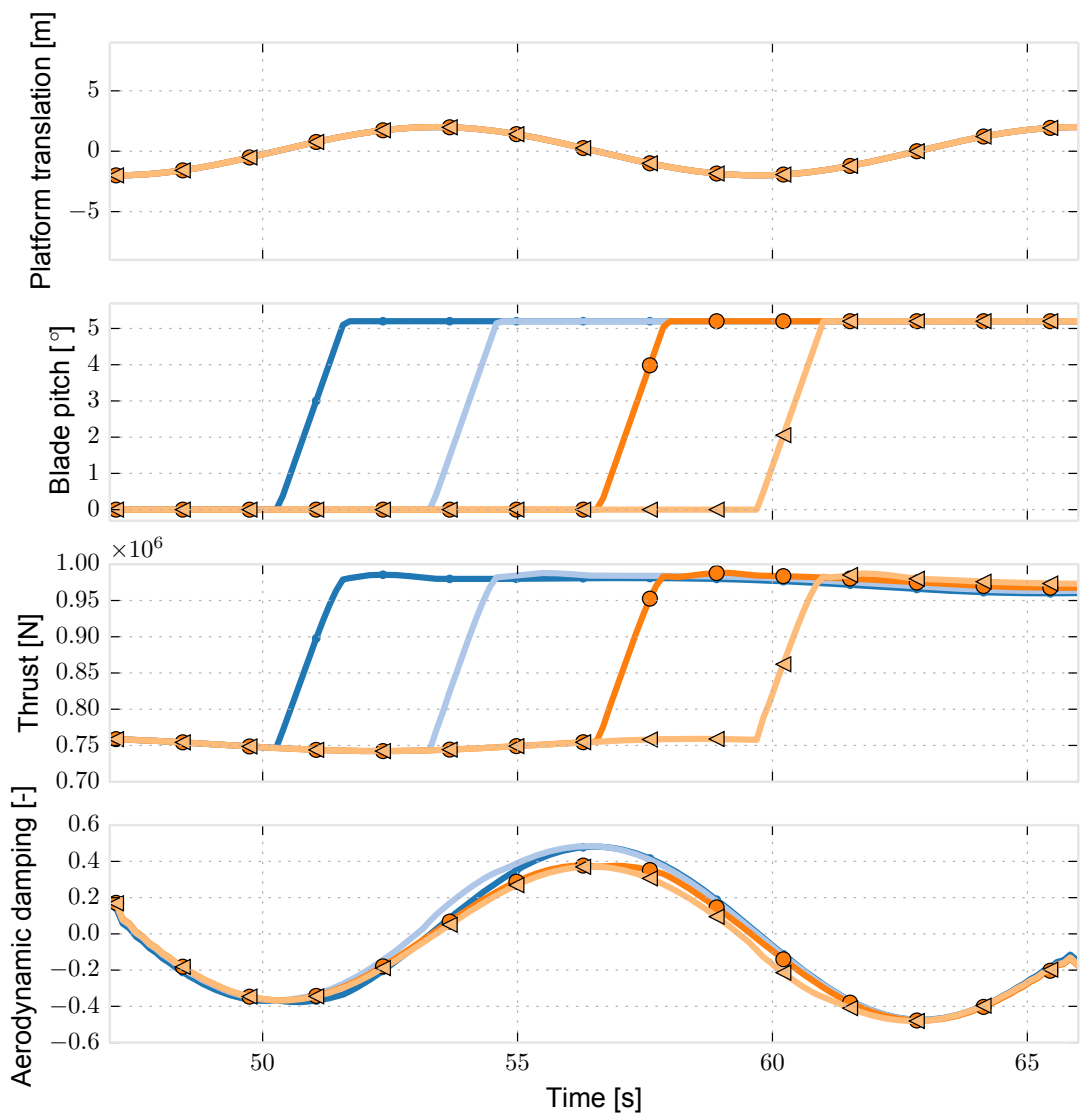

$\phi_{\text {Pitch }}=0.5 \pi-\phi_{\text {Pitch }}=\pi \bigcirc \bigcirc \phi_{\text {Pitch }}=-0.5 \pi \triangleleft \triangleleft \phi_{\text {Pitch }}=0$

Figure 9. Positive blade pitch manoeuvre.

one of the advantages of the assumptions made in the derivation. The prescribed motion of the platform must be periodic, but the thrust force response has no restriction. This in turn means that we are free to try out any control manoeuvres as long as the platform motion remains periodic.

The second demonstration case will consist of a simple $5.2^{\circ}$ blade pitch manoeuvre at a rate of $4^{\circ} \mathrm{s}^{-1}$. In total, eight cases were simulated with the pitching beginning at different phase angles with respect to the harmonic translation of the platform. The platform translation had an amplitude of $2 \mathrm{~m}$ with a frequency of $0.5 \mathrm{rad} \mathrm{s}^{-1}$. These simulation values are summarized in Table 4. The FVLLT simulation was run using the same inputs as listed earlier.

The results are presented in two sets with the negative blade pitch manoeuvre in Fig. 8 and the positive pitch manoeuvre in Fig. 9. For the negative blade pitch manoeuvres, we can see a clear increase in aerodynamic damping when the blades are pitched as the rotor retreats. Pitching the blades as the platform is advancing causes a clear drop in the aerodynamic damping. For the positive blade pitch manoeuvres, pitching as the rotor advances creates only a small increase
Table 4. Demonstration for case 2 simulation settings.

\begin{tabular}{lr}
\hline Rotor speed (rpm) & 12.1 \\
$\alpha_{0}\left(^{\circ}\right)$ & 0 \\
$\alpha_{\text {Manoeuvre }}\left(^{\circ}\right)$ & $5.2,-5.2$ \\
$\omega_{\text {pitch }}\left({ }^{\circ} \mathrm{s}^{-1}\right)$ & 4 \\
$\phi_{\text {pitch }}(-)$ & $0,0.5 \pi, \pi, 1.5 \pi$ \\
Inflow velocity $\left(\mathrm{m} \mathrm{s}^{-1}\right)$ & 11.4 \\
$\omega_{\text {platform }}\left(\mathrm{rad} \mathrm{s}^{-1}\right)$ & 0.5 \\
$X_{0}(\mathrm{~m})$ & 4 \\
\hline
\end{tabular}

in the aerodynamic damping compared to the retreating case. These kinds of results could help design a controller which restricts negative blade pitch rates as the rotor plane advances in order to maximize tower-top stability. The same approach is also useful for cases with other variations like changes in rotor speed for start up or shut down. 


\section{Conclusions}

The QBlade implementation of the lifting-line free vortex wake (LLFVW) method proved to be a useful tool for analysing floating platform wind turbines. Comparisons against two independent test cases using a variety of methods showed relatively good agreement in thrust forces, rotor power, blade forces and rotor plane induction. Along with the many verifications already undertaken in the literature, it seems that the code will perform solidly even in these challenging cases. Further work is required to extend the same analysis with flexible blades, tower and eventually platform rather than prescribed motion; research on some of these topics is already under way.

A new formulation of the instantaneous aerodynamic thrust damping of a wind turbine rotor was described. The first demonstration case was used to verify that the cycleaveraged damping values line up with well-established methods. The case also showed how the system alternated between being stable and unstable within a single cycle. The second demonstration case showed a more complicated pitch manoeuvre; the instantaneous damping method was useful in understanding the system but provided helpful information for designing control strategies. It would be useful in future work to generalize the method so that any mode shape could be analysed without having to undertake the extensive derivation described in this paper.

Code availability. The full implementation of QBlade is available for download at https://sourceforge.net/projects/qblade/.

Data availability. No additional data were generated that have not been included in this article.

Competing interests. The authors declare that they have no conflict of interest.

Special issue statement. This article is part of the special issue "The Science of Making Torque from Wind (TORQUE) 2016". It is a result of the The Science of Making Torque from Wind (TORQUE 2016), Munich, Germany, 5-7 October 2016.

Acknowledgements. We acknowledge support by the German Research Foundation and the open-access publication funds of the Technische Universität Berlin.

Edited by: Carlo L. Bottasso

Reviewed by: Vasilis A. Riziotis and two anonymous referees

\section{References}

Bisplinghoff, R. L., Ashley, H., and Halfman, R. L.: Aeroelasticity, Courier Corporation, Dover Publications, Minoela New York, 2013.

Bowles, P. O., Corke, T. C., Coleman, D. G., Thomas, F. O., and Wasikowski, M.: Improved Understanding of Aerodynamic Damping Through the Hilbert Transform, AIAA J., 52, 23842394, https://doi.org/10.2514/1.J052630, 2014.

Carta, F. O. and Niebanck, C. F.: Prediction of rotor instability at high forward speeds, Tech. rep., USAAVLABS TR, Clearinghouse for Federal Scientific and Technical Information, Springfield, Va, 1969.

Corke, T. and Thomas, F.: Dynamic Stall in Pitching Airfoils: Aerodynamic Damping and Compressibility Effects, Annu. Rev. Fluid Mech., 47, 479-505, https://doi.org/10.1146/annurevfluid-010814-013632, 2015.

Eisele, O., Pechlivanoglou, G., Nayeri, C. N., and Paschereit, C. O.: GT2013-94689 The performance of wind turbine airfoils, in: Proceedings of ASME Turbo Expo 2013, 1-10, San Antonio, 2013.

Hansen, M. O. L.: Aerodynamics of Wind Turbines, 2nd Edn., Earthscan LLC, London Sterling, VA, 2008.

Jonkman, J. M.: NREL Offshore Baseline 5MW_Onshore with Compiled Controller, available at: http://wind.nrel.gov/public/ jjonkman/NRELOffshrBsline5MW/ (last access: 1 April 2015), 2013.

Jonkman, J. M.: Fast Users Guide, available at: https://nwtc.nrel. gov/FAST7, last access: 1 April 2015.

Lennie, M., Pechlivanoglou, G., Marten, D., Nayeri, C. N., and Paschereit, C. O.: GT2015-43249: A review of wind turbine polar data and it's effect on fatigue loads simulation accuracy, Proceedings of ASME Turbo Expo 2015: Turbine Technical Conference and Exposition GT2015, https://doi.org/10.1115/GT201543249, 2015.

Lennie, M., Bach, A., Pechlivanoglou, G., Nayeri, C., and Paschereit, C. O.: The Unsteady Aerodynamic Response of an Airfoil with Microtabs and it's Implications for Aerodynamic Damping, 34th Wind Energy Symposium, 1-12, https://doi.org/10.2514/6.2016-1006, 2016.

Marten, D., Lennie, M., Pechlivanoglou, G., Nayeri, C. N., and Paschereit, C. O.: Implementation, Optimization, and Validation of a Nonlinear Lifting Line-Free Vortex Wake Module Within the Wind Turbine Simulation Code QBlade, Journal of Engineering for Gas Turbines and Power, 138, 072601, https://doi.org/10.1115/1.4031872, 2015.

Müller-Vahl, H. F.: Wind Turbine Blade Dynamic Stall and its Control, Doctoral thesis, Technische Universität Berlin, 2015.

Pitt, D. M. and Peters, D. A.: Rotor Dynamic Inflow Derivatives and Time Constants from Various Inflow Models, in: 9th European Rotorcraft Forum, 1-24, US Army Troop Support and Aviation Materiel Readiness Command, St. Louis Missouri, 1983.

Rumsey, C. L.: Turbulence Model Verification and Validation, in: ASME Verification and Validation Symposium, NASA Langley Research Center, Las Vegas, 2016.

Saverin, J., Marten, D., Pechlivanoglou, G., Nayeri, C. N., and Paschereit, C. O.: GT2016-56290 Coupling of an unsteady lifting line free vortex wake code to the aeroelastic HAWT simulation 
suite FAST, in: Proceedings of ASME Turbo Expo 2016, Seoul, South Korea, 2016.

Scanlan, R. H. and Rosenbaum, R.: Introduction to the study of aircraft vibration and flutter, Dover Publications, New York, 1951.

Sebastian, T. and Lackner, M. A.: Characterization of the unsteady aerodynamics of offshore floating wind turbines, 16, 339-352, https://doi.org/10.1002/we.545, 2013.

Strangfeld, C.: Active control of trailing vortices by means of longand short-wavelength actuation, Doctor thesis, Technical University of Berlin, 2015.

Stangfeld, C., Rumsey, C. L., Mueller-Vahl, H., Greenblatt, D., Nayeri, C., and Paschereit, C. O.: Unsteady Thick Airfoil Aerodynamics: Experiments, Computation, and Theory, 45th AIAA Fluid Dynamics Conference, 1-19, https://doi.org/10.2514/6.2015-3071, 2015.
Theodorsen, T.: General theory of aerodynamic instability and the mechanism of flutter, available at: https://ntrs.nasa.gov/search. jsp?R=19930090935 (last access: 10 December 2017), 1935.

Tran, T., Kim, D., and Song, J.: Computational Fluid Dynamic Analysis of a Floating Offshore Wind Turbine Experiencing Platform Pitching Motion, Energies, 7, 5011-5026, https://doi.org/10.3390/en7085011, 2014.

Vaal, J. B. D., Hansen, M. O. L., and Moan, T.: Effect of wind turbine surge motion on rotor thrust, 17, 105-121, https://doi.org/10.1002/we.1562, 2014.

Wendler, J., Marten, D., Nayeri, C. N., Pechlivanoglou, G., and Paschereit, C. O.: Gt2016-57184 Implementation and Validation of an Unsteady Aerodynamics Model, in: Proceedings of ASME Turbo Expo 2016: Turbomachinery Technical Conference and Exposition, 1-10, 2016. 